\title{
ELECTRON-ION RECOMBINATION OF Fe x FORMING Fe IX AND OF Fe XI FORMING Fe x: LABORATORY MEASUREMENTS AND THEORETICAL CALCULATIONS
}

\author{
M. Lestinsky ${ }^{1,2}$, N. R. BAdnell ${ }^{3}$, D. Bernhardt ${ }^{4}$, M. Grieser ${ }^{2}$, J. HoffMAnN ${ }^{2}$, D. Lukić ${ }^{1,5}$, A. MÜller ${ }^{3}$, D. A. Orlov ${ }^{2}$, \\ R. Repnow ${ }^{2}$, D. W. SAvin ${ }^{1}$, E. W. Schmidt ${ }^{4}$, M. Schnell ${ }^{2}$, S. Schippers ${ }^{4}$, A. Wolf ${ }^{2}$, And D. Yu ${ }^{4,6}$ \\ ${ }^{1}$ Columbia Astrophysics Laboratory, Columbia University, New York, NY 10027, USA; lestinsky@ astro.columbia.edu \\ ${ }^{2}$ Max-Planck-Institute for Nuclear Physics, 69117 Heidelberg, Germany \\ ${ }^{3}$ Department of Physics, University of Strathclyde, Glasgow G4 0NG, UK \\ ${ }^{4}$ Institut für Atom- und Molekülphysik, Justus-Liebig-Universität Giessen, D-35392 Giessen, Germany \\ Received 2009 January 15; accepted 2009 April 1; published 2009 May 22
}

\begin{abstract}
We have measured electron-ion recombination for $\mathrm{Fe}^{9+}$ forming $\mathrm{Fe}^{8+}$ and for $\mathrm{Fe}^{10+}$ forming $\mathrm{Fe}^{9+}$ using a merged beams arrangement at the TSR heavy-ion storage ring in Heidelberg, Germany. The measured merged beams recombination rate coefficients (MBRRC) for relative energies from 0 to $75 \mathrm{eV}$ are presented, covering all dielectronic recombination (DR) resonances associated with $3 s \rightarrow 3 p$ and $3 p \rightarrow 3 d$ core transitions in the spectroscopic species $\mathrm{Fe} \mathrm{x}$ and $\mathrm{Fe} \mathrm{XI}$, respectively. We compare our experimental results to state-ofthe-art multiconfiguration Breit-Pauli (MCBP) calculations and find significant differences. Poor agreement between the measured and theoretical resonance structure is seen for collision energies below $48 \mathrm{eV}$ for $\mathrm{Fe} \mathrm{x}$ and below $35 \mathrm{eV}$ for Fe XI. The integrated resonance strengths, though, are in reasonable agreement. At higher energies, good agreement is seen for the resonance structure but for the resonance strengths theory is significantly larger than experiment by a factor of $\approx 1.5(2)$ for $\mathrm{Fe} x(\mathrm{Fe} \mathrm{xI})$. From the measured MBRRC, we have extracted the DR contributions and transform them into plasma recombination rate coefficients (PRRCs) for astrophysical plasmas with temperatures in the range of $10^{2}-10^{7} \mathrm{~K}$. This range spans across the regimes where each ion forms in photoionized or in collisionally ionized plasmas. For both temperature regimes, the experimental uncertainties are $25 \%$ at a $90 \%$ confidence level. As expected based on predictions from active galactic nucleus observations as well as our previous laboratory and theoretical work on M-shell iron, the formerly recommended DR data severely underestimated the rate coefficient at temperatures relevant for photoionized gas. At these temperatures relevant for photoionized gas, we find agreement between our experimental results and MCBP theory. This is somewhat surprising given the poor agreement in MBRRC resonance structure. At the higher temperatures relevant for collisionally ionized gas, the MCBP calculations yield an Fe XI DR rate coefficient that is significantly larger than the experimentally derived one. We present parameterized fits to our experimentally derived DR PRRC for ease of inclusion into astrophysical modeling codes.
\end{abstract}

Key words: atomic data - atomic processes - galaxies: active - galaxies: nuclei - plasmas - X-rays: galaxies

\section{INTRODUCTION}

Recent Chandra and XMM-Newton X-ray observations of active galactic nuclei (AGNs) have detected a new absorption feature in the 15-17 $\AA$ wavelength range. This has been identified as an unresolved transition array (UTA) due mainly to $2 p \rightarrow 3 d$ inner shell absorption in iron ions with an open $\mathrm{M}$ shell (Fe I-XVI). This spectral feature is believed to originate in the warm absorber material surrounding the central supermassive black hole in AGNs. It has been identified in a number of AGNs including IRAS 13349+2438 (Sako et al. 2001), Mrk 509 (Pounds et al. 2001), NGC 3783 (Blustin et al. 2002; Kaspi et al. 2002; Behar et al. 2003), MCG -6-30-15 and Mrk 766 (Sako et al. 2003), NGC 5548 (Steenbrugge et al. 2003), I $\mathrm{Zw} 1$ (Gallo et al. 2004), MR 2251-178 (Kaspi et al. 2004), Akn 564 (Matsumoto et al. 2004), NGC 4051 (Pounds et al. 2004), NGC 985 (Krongold et al. 2005), IC 4329A (Steenbrugge et al. 2005), and NGC 3516 (McKernan et al. 2007).

Spectra from AGNs are typically analyzed using publicly available photoionization codes such as Cloudy (Ferland et al. 1998) and XSTAR (Kallman \& Bautista 2001; Kallman et al. 2004). The modeled temperature and ionization balance of

\footnotetext{
5 Current address: Institute of Physics, Belgrade, 11080 Zemun, Serbia.

6 Permanent address: Institute of Modern Physics, Chinese Academy of Sciences, Lanzhou 730000, People's Republic of China.
}

photoionized gas depend on the ionization parameter which is the ratio of the radiation field strength to the gas density. A number of different definitions exist for this parameter. Here, we use $\xi=L / n R^{2}$, where $L$ is the luminosity of the radiation source, $n$ is the gas density, and $R$ is the distance to the source.

Based on atomic structure calculations and photoabsorption modeling, Behar et al. (2001) have shown that the shapes, central wavelengths, and equivalent widths of the UTAs can be used to diagnose the properties of AGN warm absorbers. However, models which match absorption features from secondand third-row elements cannot correctly reproduce the observed UTAs from the fourth-row element iron. At the value of $\xi$ inferred from the second- and third-row elements, the models appear to predict too high an ionization level for iron, as was noted for NGC 3783 by Netzer et al. (2003). They attributed this discrepancy to an underestimate of the low-temperature dielectronic recombination (DR) rate coefficients for the Fe Mshell ions used in the models. To investigate this possibility, Netzer (2004) and Kraemer et al. (2004) arbitrarily increased the low-temperature Fe M-shell DR rate coefficients. Their model results obtained with the modified DR rate coefficients support the hypothesis of Netzer et al. (2003).

These recent AGN observations have motivated experimental studies of low-temperature DR for Fe M-shell ions (Schmidt et al. 2006, 2008; Lukić et al. 2007) and theoretical studies for Na- through Ar-like iron (Gu 2004; Badnell 2006a, 2006b; 
Altun et al. 2006, 2007). Here and throughout this paper, the recombining systems are identified by their initial charge state. In the temperature ranges where these ions form in photoionized plasmas (PPs), these new Fe M-shell DR data are up to orders of magnitude larger than the DR data previously available. They are even significantly larger than the ad hoc modified DR rate coefficients of Netzer (2004) and Kraemer et al. (2004). These new Fe M-shell DR data appear to reduce the discrepancies involving the Fe M-shell UTA issues noted above.

Spectral fits to NGC 3783 using the DR data available before $\sim 2006$ yield multiphase models with a $\log (\xi) \sim 2 \mathrm{erg} \mathrm{cm} \mathrm{s}^{-1}$ component to account for absorption features from second- and third-row elements and $\log (\xi) \sim 0$ erg $\mathrm{cm} \mathrm{s}^{-1}$ component to account for the Fe M-shell UTA (e.g., McKernan et al. 2007; T. R. Kallman 2008, private communication). Fits using the DR data available after $\sim 2006$ find little change in $\log (\xi)$ derived from the second- and third-row elements. However, using these later DR data, the bulk of the Na- through Ar-like Fe ions form at $\operatorname{ag} \log (\xi)$ which is closer to the value inferred from absorption lines in the spectrum due to the second- and third-row elements (T. R. Kallman 2008, private communication).

DR also plays an important role in determining the thermal stability of AGN warm absorbers. It has long been known that such gas is thermally unstable at temperatures of $\sim 10^{5} \mathrm{~K}$ (Krolik et al. 1981; Reynolds \& Fabian 1995; Hess et al. 1997; Chakravorty et al. 2008). Hess et al. (1997) showed that Fe Lshell ions play an important role in determining the range in parameter space over which photoionized gas is predicted to be thermally unstable. This instability is robust to changes in elemental abundances, shape of the ionizing spectrum, particle density, and optical depth (Reynolds \& Fabian 1995; Hess et al. 1997). Savin et al. (1999) explored how uncertainties in the Fe L-shell ion DR data available at that time affected the predicted instability and found that varying the DR rate coefficients dramatically changed the range in parameter space over which the gas was unstable. Attempts to understand this instability motivated a series of Fe L-shell DR measurements for F-like Fe XVIII (Savin et al. 1997, 1999), O-like Fe XIX (Savin et al. 1999, 2002b), N-like Fe xx (Savin et al. 2002a), C-like Fe XXI and B-like Fe XXII (Savin et al. 2003), and Belike Fe XXIII (Savin et al. 2006). Our experimental work also motivated a series of state-of-the-art DR calculations for L-shell ions (Badnell et al. 2003; Gu 2003).

Recently, Chakravorty et al. (2008) have used the state-ofthe-art theoretical DR data of Badnell et al. (2003) and Badnell (2006a, 2006b) to re-investigate this thermal instability. They find that the new Fe L- and M-shell ion DR data result in "a larger probability of having a thermally stable warm absorber at $10^{5} \mathrm{~K}$ than previous predictions and also a greater possibility for multiphase gas."

The reliability of the analyses reported above for the Fe Mshell UTAs and for the thermal stability of warm absorbers at $\sim 10^{5} \mathrm{~K}$ depends on the accuracy of the DR data used. However, the two theorists responsible for much of the available state-ofthe-art theoretical DR rate coefficients estimate that were they to carry out calculations for M-shell iron ions in charge stages $6+$ and lower, then such data can be uncertain by up to a factor of 10 due to uncertainties in the position of the relevant low-energy DR resonances (N. R. Badnell 2008, private communication; M. F. Gu 2008, private communication).

In response to the clear need for reliable low-temperature $\mathrm{Fe}$ M-shell DR data, we are conducting a series of ion storagering merged beams measurements. These are being carried out utilizing the TSR heavy-ion storage ring (Habs et al. 1989) located at the Max-Planck-Institute for Nuclear Physics in Heidelberg, Germany. Using TSR we have already carried out DR measurements for Mg-like Fe XV (Lukić et al. 2007), Allike Fe XIV (Schmidt et al. 2006), and Ar-like Fe IX and K-like Fe VIII (Schmidt et al. 2008). Work predating the current project on Na-like Fe XVI was carried out by Linkemann et al. (1995) and Müller (1999). A bibliographic compilation of storage-ring DR measurements with astrophysically relevant ions has recently been given by Schippers (2008).

DR is a two-step recombination process that begins when a free electron approaches an ion, collisionally excites a bound electron of the ion, and is simultaneously captured into a level with principle quantum number $n$. The electron excitation can be labeled $N l_{j} \rightarrow N^{\prime} l_{j^{\prime}}^{\prime}$, where $N$ is the principal quantum number of the core electron, $l$ is the orbital angular momentum, and $j$ is its total angular momentum. Conservation of energy requires for dielectronic capture that

$$
\Delta E=E_{k}+E_{b},
$$

where $\Delta E$ is the core excitation energy, $E_{k}$ is the kinetic energy of the incident electron, and $E_{b}$ is the binding energy of the captured electron in the core-excited ion. Because $\Delta E$ and $E_{b}$ are quantized, DR is a resonant process. We classify the core excitation by $\Delta N=N^{\prime}-N$. The intermediate state, formed by simultaneous excitation and capture, may autoionize. The DR process is complete when the intermediate state emits a photon which reduces the total energy of the recombined ion to below its ionization limit. See Müller (2008) for additional details.

In this paper, we report our recent results for measurements of $\Delta N=0 \mathrm{DR}$ in Cl-like Fe $\mathrm{x}$ and S-like Fe XI and derive plasma rate coefficients for inclusion in astrophysical modeling codes. The relevant channels for Fe $\mathrm{x}$ are

$$
\mathrm{Fe}^{9+}\left(3 s^{2} 3 p^{5}\left[{ }^{2} P_{3 / 2}^{o}\right]\right)+\mathrm{e}^{-} \rightarrow\left\{\begin{array}{l}
\mathrm{Fe}^{8+}\left(3 s^{2} 3 p^{5}\left[^{2} P_{1 / 2}^{o}\right] n l\right) \\
\mathrm{Fe}^{8+}\left(3 s 3 p^{6} n l\right) \\
\mathrm{Fe}^{8+}\left(3 s^{2} 3 p^{4} 3 d n l\right) \\
\mathrm{Fe}^{8+}\left(3 s 3 p^{5} 3 d n l\right) .
\end{array}\right.
$$

For the Fe XI parent ion, the relevant capture channels are

$$
\mathrm{Fe}^{10+}\left(3 s^{2} 3 p^{4}\left[{ }^{3} P_{2}\right]\right)+\mathrm{e}^{-} \rightarrow\left\{\begin{array}{l}
\mathrm{Fe}^{9+}\left(3 s^{2} 3 p^{4}\left[{ }^{3} P_{1,0} ;{ }^{1} D_{2} ;{ }^{1} S_{0}\right] n l\right) \\
\mathrm{Fe}^{9+}\left(3 s 3 p^{5} n l\right) \\
\mathrm{Fe}^{9+}\left(3 s^{2} 3 p^{3} 3 d n l\right) \\
\mathrm{Fe}^{9+}\left(3 s 3 p^{4} 3 d n l\right) .
\end{array}\right.
$$

In the energy range below approximately $90(80) \mathrm{eV}$, the DR spectrum for $\mathrm{Fe} \mathrm{x}(\mathrm{Fe} \mathrm{XI})$ is dominated by $\Delta N=0$ core excitations. The energies of the core transitions relative to the ground levels of the respective parent ions are listed in Tables 1 and 2 .

The remainder of this paper is organized as follows: Section 2 gives a brief summary of the theoretical description. Section 3 describes the experimental setup used in this work. Section 4 shows our experimental results for the merged beams recombination rate coefficient (MBRRC) for S-like Fe XI and Cl-like Fe $\mathrm{x}$. The experimentally derived DR plasma recombination rate coefficient (PRRC), a comparison with theory, and fitting results for each ion are also presented in Section 5. A summary is given in Section 6. 
Table 1

Energy Levels of Fe $\mathrm{x}$ Relative to the $3 s^{2} 3 p^{5}\left[{ }^{2} \mathrm{P}_{3 / 2}^{o}\right]$ Ground Level (Ralchenko et al. 2006) for Excitations Within the M shell

\begin{tabular}{lc}
\hline \hline Level & Energy (eV) \\
\hline $3 s^{2} 3 p^{5}\left[{ }^{2} P_{1 / 2}^{o}\right]$ & 1.94446 \\
$3 s 3 p^{6}\left[{ }^{2} S_{1 / 2}\right]$ & 35.8623 \\
$3 s^{2} 3 p^{4}\left({ }^{3} P\right) 3 d\left[{ }^{4} D_{7 / 2}\right]$ & 48.1938 \\
$3 s^{2} 3 p^{4}\left({ }^{3} P\right) 3 d\left[{ }^{4} D_{5 / 2}\right]$ & 48.1938 \\
$3 s^{2} 3 p^{4}\left({ }^{3} P\right) 3 d\left[{ }^{4} D_{3 / 2}\right]$ & 48.3600 \\
$3 s^{2} 3 p^{4}\left({ }^{3} P\right) 3 d\left[{ }^{4} D_{1 / 2}\right]$ & 48.5466 \\
$3 s^{2} 3 p^{4}\left({ }^{3} P\right) 3 d\left[{ }^{4} F_{9 / 2}\right]$ & 51.7824 \\
$3 s^{2} 3 p^{4}\left({ }^{3} P\right) 3 d\left[{ }^{4} F_{7 / 2}\right]$ & 52.4199 \\
$3 s^{2} 3 p^{4}\left({ }^{3} P\right) 3 d\left[{ }^{4} F_{5 / 2}\right]$ & 52.9119 \\
$3 s^{2} 3 p^{4}\left({ }^{3} P\right) 3 d\left[{ }^{4} F_{3 / 2}\right]$ & 53.1022 \\
$3 s^{2} 3 p^{4}\left({ }^{1} D\right) 3 d\left[{ }^{2} P_{3 / 2}\right]$ & 53.5522 \\
$3 s^{2} 3 p^{4}\left({ }^{1} D\right) 3 d\left[{ }^{2} D_{3 / 2}\right]$ & 53.8853 \\
$3 s^{2} 3 p^{4}\left({ }^{3} P\right) 3 d\left[{ }^{4} P_{1 / 2}\right]$ & 53.9083 \\
$3 s^{2} 3 p^{4}\left({ }^{3} P\right) 3 d\left[{ }^{4} P_{5 / 2}\right]$ & 54.7828 \\
$3 s^{2} 3 p^{4}\left({ }^{3} P\right) 3 d\left[{ }^{2} F_{7 / 2}\right]$ & 54.6572 \\
$3 s^{2} 3 p^{4}\left({ }^{3} P\right) 3 d\left[{ }^{2} F_{5 / 2}\right]$ & $56.1314^{\mathrm{a}}$ \\
$3 s^{2} 3 p^{4}\left({ }^{1} D\right) 3 d\left[{ }^{2} G_{9 / 2}\right]$ & 55.8860 \\
$3 s^{2} 3 p^{4}\left({ }^{1} D\right) 3 d\left[{ }^{2} G_{7 / 2}\right]$ & 55.9273 \\
$3 s^{2} 3 p^{4}\left({ }^{1} D\right) 3 d\left[{ }^{2} F_{5 / 2}\right]$ & $59.1031^{\mathrm{a}}$ \\
$3 s^{2} 3 p^{4}\left({ }^{1} D\right) 3 d\left[{ }^{2} F_{7 / 2}\right]$ & 60.2542 \\
$3 s^{2} 3 p^{4}\left({ }^{1} S\right) 3 d\left[\left[^{2} D_{5 / 2}\right]\right.$ & $\ldots . \mathrm{b}$ \\
$3 s^{2} 3 p^{4}\left({ }^{1} S\right) 3 d\left[{ }^{2} D_{3 / 2}\right]$ & 63.4551 \\
$3 s^{2} 3 p^{4}\left({ }^{1} D\right) 3 d\left[{ }^{2} S_{1 / 2}\right]$ & 67.1844 \\
$3 s^{2} 3 p^{4}\left({ }^{3} P\right) 3 d\left[{ }^{2} P_{3 / 2}\right]$ & 69.9516 \\
$3 s^{2} 3 p^{4}\left({ }^{3} P\right) 3 d\left[{ }^{2} P_{1 / 2}\right]$ & 70.6691 \\
$3 s^{2} 3 p^{4}\left({ }^{3} P\right) 3 d\left[{ }^{2} D_{5 / 2}\right]$ & 71.0372 \\
$3 s^{2} 3 p^{4}\left({ }^{3} P\right) 3 d\left[{ }^{2} D_{3 / 2}\right]$ & 72.6850 \\
$3 s 3 p^{5}\left({ }^{3} P^{o}\right) 3 d\left[{ }^{4} F_{9 / 2}^{o}\right]$ & 86.3750 \\
$3 s 3 p^{5}\left({ }^{3} P^{o}\right) 3 d\left[{ }^{4} F_{7 / 2}^{o}\right]$ & 86.7259 \\
$3 s 3 p^{5}\left({ }^{3} P^{o}\right) 3 d\left[{ }^{4} F_{5 / 2}^{o}\right]$ & 87.1094 \\
$3 s 3 p^{5}\left({ }^{3} P^{o}\right) 3 d\left[{ }^{4} F_{3 / 2}^{o}\right]$ & 87.4622 \\
\hline
\end{tabular}

Notes. We are unaware of any published data for any of the excited core levels not listed here.

a According to Ralchenko et al. (2006), this level may not be real.

b Value not specified by Ralchenko et al. (2006).

\section{THEORY}

Jacobs et al. (1977) published the first DR calculations for Fe $\mathrm{X}$ and Fe XI using a "no-coupling" scheme, allowing only for dipole core excitations in the dielectronic capture process and paying no detailed attention to the low-energy resonances important in photoionized gas. This was because their work was intended for high-temperature plasmas and computational limitations of the time necessitated truncating the calculations to keep them tractable. Arnaud \& Raymond (1992) incorporated the Jacobs et al. (1977) results and adjusted them to the hightemperature behavior of the spin-orbit coupling calculations from Hahn (1989).

Stimulated by the AGN X-ray observations cited in Section 1 and by our experimental results for a number of $\mathrm{M}$-shell iron ions, Badnell (2006b) revisited a series of Fe M-shell ions, including the $\mathrm{Fe} \mathrm{x}$ and $\mathrm{Fe} \mathrm{XI}$ ions. Using the AUTOSTRUCTURE package (Badnell 1986), he performed multiconfiguration Breit-Pauli (MCBP) calculations for Fe ions with valence $3 p^{q}$ electrons $(q=0-6)$. In this state-of-the-art theory, photorecombination, that is radiative recombination (RR) and DR, is calculated in the independent process isolated resonance approximation (Seaton \& Storey 1976), i.e., RR is treated separately from DR. The validity of this approach has been demon-
Table 2

Same as Table 1, but for Fe XI Relative to the $3 s^{2} 3 p^{4}\left[{ }^{3} P_{2}\right]$ Ground State

\begin{tabular}{lc}
\hline \hline Level & Energy $(\mathrm{eV})$ \\
\hline $3 s^{2} 3 p^{4}\left[{ }^{3} P_{1}\right]$ & 1.57062 \\
$3 s^{2} 3 p^{4}\left[{ }^{3} P_{0}\right]$ & 1.7745 \\
$3 s^{2} 3 p^{4}\left[{ }^{1} D_{2}\right]$ & 4.67961 \\
$3 s^{2} 3 p^{4}\left[{ }^{1} S_{0}\right]$ & 10.0197 \\
$3 s 3 p^{5}\left[{ }^{3} P_{2}^{o}\right]$ & 35.1567 \\
$3 s 3 p^{5}\left[{ }^{3} P_{1}^{o}\right]$ & 36.3470 \\
$3 s 3 p^{5}\left[{ }^{3} P_{0}^{o}\right]$ & 37.0915 \\
$3 s 3 p^{5}\left[{ }^{1} P_{1}^{o}\right]$ & 44.8627 \\
$3 s^{2} 3 p^{3}\left({ }^{2} P^{o}\right) 3 d\left[{ }^{3} P_{2}^{o}\right]$ & 61.5073 \\
$3 s^{2} 3 p^{3}\left({ }^{2} D^{o}\right) 3 d\left[{ }^{3} S_{1}^{o}\right]$ & 65.2752 \\
$3 s^{2} 3 p^{3}\left({ }^{2} D^{o}\right) 3 d\left[{ }^{3} P_{2}^{o}\right]$ & 65.8716 \\
$3 s^{2} 3 p^{3}\left({ }^{2} D^{o}\right) 3 d\left[{ }^{3} P_{1}^{o}\right]$ & 67.1238 \\
$3 s^{2} 3 p^{3}\left({ }^{2} D^{o}\right) 3 d\left[{ }^{3} P_{0}^{o}\right]$ & 67.1647 \\
$3 s^{2} 3 p^{3}\left({ }^{2} D^{o}\right) 3 d\left[{ }^{1} P_{1}^{o}\right]$ & 66.1394 \\
$3 s^{2} 3 p^{3}\left({ }^{4} S^{o}\right) 3 d\left[{ }^{3} D_{3}^{o}\right]$ & 68.7244 \\
$3 s^{2} 3 p^{3}\left({ }^{4} S^{o}\right) 3 d\left[{ }^{3} D_{2}^{o}\right]$ & 69.6308 \\
$3 s^{2} 3 p^{3}\left({ }^{4} S^{o}\right) 3 d\left[{ }^{3} D_{1}^{o}\right]$ & 70.2222 \\
$3 s^{2} 3 p^{3}\left({ }^{2} D^{o}\right) 3 d\left[{ }^{1} D_{2}^{o}\right]$ & 71.7695 \\
$3 s^{2} 3 p^{3}\left({ }^{2} D^{o}\right) 3 d\left[{ }^{1} F_{3}^{o}\right]$ & 73.6503 \\
$3 s^{2} 3 p^{3}\left({ }^{2} P^{o}\right) 3 d\left[{ }^{1} P_{1}^{o}\right]$ & 77.2521 \\
\hline
\end{tabular}

strated by Pindzola et al. (1992). Note that it should be implicitly understood throughout that DR also comprises trielectronic recombination (TR) and possible higher order resonant processes wherever these are significant. The importance of TR has been shown by Schnell et al. (2003) in Be-like Cl XIV, where TR contributes up to $40 \%$ of the photorecombination plasma rate coefficient, depending on the plasma temperature.

Details of the MCBP DR calculations have been reported in Badnell et al. (2003). Briefly, the AUTOSTRUCTURE code was used to calculate energy levels as well as radiative and autoionization rates in the intermediate coupling approximation. These must be postprocessed to obtain the final-state, level-resolved, and total DR data. The energy levels were shifted to known spectroscopic values for the $3 \rightarrow 3$ transitions. Radiative transitions between autoionizing states were accounted for in the calculation. The DR cross section was approximated by the sum of Lorentzian profiles for all included resonances. The basic configurations which describe the $N$-electron target for $\mathrm{Fe} \mathrm{x}$ are $3 s^{2} 3 p^{5}, 3 s 3 p^{6}, 3 s^{2} 3 p^{4} 3 d, 3 s 3 p^{5} 3 d, 3 s^{2} 3 p^{3} 3 d^{2}$, and $3 s^{0} 3 p^{6} 3 d$. The Fe XI target configurations are $3 s^{2} 3 p^{4}, 3 s 3 p^{5}$, $3 s^{2} 3 p^{3} 3 d, 3 p^{6}, 3 s 3 p^{4} 3 d, 3 s^{2} 3 p^{2} 3 d^{2}$, and $3 s^{0} 3 p^{5} 3 d$. A closed-shell Ne-like core is assumed for each ion. Both configuration sets are identified as "7CF" in Badnell (2006b).

For $\mathrm{Fe} \mathrm{X}$, we derived a theoretical merged beams DR rate coefficient by reproducing the earlier calculations of Badnell (2006b). For Fe XI, we have carried out new DR calculations using the state-of-the-art MCBP theoretical method as described in Badnell (2006b). In both calculations only the strong $\Delta N=0$ DR channels associated with $3 \rightarrow 3$ transitions have been considered. The weaker $\Delta N \geqslant 1 \mathrm{DR}$ channels have been neglected in order to keep the calculation manageable. For the $3 p^{q}$ systems considered by Badnell (2006b), their contributions to the DR PRRC have been estimated to be insignificant for PPs and less than $10 \%$ of the $\Delta N=0$ rate coefficient for collisionally ionized plasmas (CPs).

Revisiting the $\mathrm{Fe}$ XI calculations performed in Badnell (2006b) turned out to be necessary as initially the theoretical energy structure of the Rydberg series limits at around $65 \mathrm{eV}$ did not coincide with the experimental results. Badnell (2006b) 
calculated the levels of core excitations for all configurations in the "7CF" list. Those results were shifted to match the tabulated level data of Ralchenko et al. (2006). However, for the Fe XI $3 s^{2} 3 p^{3} 3 d$ configuration, the tabulated levels are incomplete and Badnell (2006b) shifted all of the roughly 50 levels of this configuration by the average shift derived from the 12 tabulated levels. Here, we shift these 12 known levels to their explicit positions given by Ralchenko et al. (2006) and use the average shift only for the remaining levels. This improves the agreement between theory and experiment for the energy structure at the $3 s^{2} 3 p^{3} 3 d$ series limits. The resulting new theoretical plasma rate coefficient changes by less than $3 \%$ from the Badnell (2006b) data in the regimes where Fe XI is abundant in photoionized or CPs.

RR calculations for iron M-shell ions were also carried out by Badnell (2006b) using AUTOSTRUCTURE. Here, we use his RR cross section results for both Fe $\mathrm{X}$ and Fe XI.

\section{EXPERIMENTAL SETUP}

Measurements were performed utilizing the heavy-ion storage-ring TSR. The experimental method used has been described in detail by Kilgus et al. (1992), Lampert et al. (1996), Pastuszka et al. (1996), Schippers et al. (2001), Wolf et al. (2006), Lestinsky et al. (2008), and Schmidt et al. (2008). The key components are discussed briefly here. See, in particular, Schmidt et al. (2008) for details not touched on here.

The TSR facility is uniquely configured with two separate electron-ion merged beams sections. This instrumentation allows us to perform continuous electron cooling (Poth 1990) in one straight section and to carry out measurements of the desired electron-ion collisions in another section. The first electron beam apparatus merges a magnetically guided cold electron beam with the ions for a distance of $\approx 1.5 \mathrm{~m}$. This copropagating electron beam serves as a heat bath for the ions (hereafter, the electron cooler; Steck et al. 1990). The second apparatus operates as a dedicated collisional probe for measurements at tunable relative velocity (hereafter, the electron target or probe beam; Sprenger et al. 2004). It employs schemes for adiabatic transverse expansion and adiabatic acceleration to achieve low transverse and longitudinal temperature spreads of the probe beam.

The physical separation of the cooling and probe beam functions also has the benefits of improving the ion beam quality by allowing for constant cooling of the ions. Simultaneous cooling while probing stabilizes the ion beam energy. The drag force (Wolf et al. 2006) exerted on the ion beam by the probing electron beam is offset by the cooling forces action in the ions in the cooler. This improves the achievable resolution as well as the precision of the energy scale at low relative energies.

In the recombination process, the momentum transfer is small and the recombined ions insignificantly alter their initial trajectory. However, due to their decreased charge state, they are deflected by the first dipole magnet downstream of the electron target onto a trajectory different from the parent ions and directed onto a YAP:Ce crystal (Baryshevsky et al. 1991) used as a fast scintillator which can be positioned within the vacuum system of TSR to collect a desired product ion species (Wissler 2002; Lestinsky 2007). The impacting ions deposit their complete kinetic energy in the crystal and stimulate the emission of hundreds of photons within a few nanoseconds. These light pulses are coupled through a window onto a photomultiplier tube, and its output signal is amplified and discriminated. The discriminator output pulses are counted in a scaler channel of the data acquisition computer controlling the experiment. By stepping the detector position horizontally and vertically through the recombined ion beam, the width of the product beam was determined to $\approx 1 \mathrm{~mm}$ in each direction, which basically is an image of the stored cooled parent beam. With a detector size of $20 \mathrm{~mm} \times 20 \mathrm{~mm}$, all recombined ions created in the electron-ion overlap region are collected by the active detector surface after aligning the center of the detector to the center of the product beam. The detector gives a signal which consists almost entirely of recombined target ions; cosmic ray incidents are observed with a rate of $<1 \mathrm{~Hz}$. With a pulse height spread of $\approx 5 \%$, the discriminator cutoff can be set safely without suppressing real events. The total count rate was less than $100 \mathrm{kHz}$ at all times. The dead time of the detector was determined by the discriminator pulse length of $50 \mathrm{~ns}$. Thus, we assume an upper limit for dead-time induced loss of events to below $0.5 \%$. Hence, we can exclude electronic or geometric cutoffs to the detection efficiency and assume a detector efficiency of unity.

Along the trajectory from the electron-ion interaction region to the detector, the recombined ions experience various magnetic fields, e.g., due to the demerging section of the electron target, focusing elements in the TSR beamline, and the first dipole magnet downstream of the target. These magnetic fields induce motional electric fields as seen by the ions, leading to field ionization of high- $n$ Rydberg levels. For the magnetic field strengths and the ion velocities used, the semiclassical value of this cutoff (Gallagher 1994) is $n_{\text {cut }}=39$ for Fe x and 41 for Fe XI. However, some of the $n$ levels above $n_{\text {cut }}$ can radiatively decay to below $n_{\text {cut }}$ during the flight time from the target through the magnets and are thus detected. These ionization and radiative de-excitation processes have been described in detail by Schippers et al. (2001), and are quantitatively well understood with $(n, l)$-specific modeling of the de-excitation probabilities. We use this approach when comparing our MCBP results to our experimental results.

Data collection was performed by repeating a measurement cycle consisting of successive phases of injection, electron cooling, and energy scans. For the present work, $\mathrm{Fe}^{9+}$ and $\mathrm{Fe}^{10+}$ beams of 93.2 and $115 \mathrm{MeV}$, respectively, were prepared in a tandem accelerator, charge and mass selected (atomic weight $A=56$ ), and injected into TSR using multiturn injection and ecool stacking (Grieser et al. 1991). During the injection phase, ions were fed into TSR in pulses once per second. Simultaneous electron cooling decreased the spatial and velocity spreads of newly injected ions, freeing up storage-ring acceptance phase space volume for the following injection shot. Accumulation of ions over 13 and 10 injections were required per cycle for $\mathrm{Fe}^{9+}$ and $\mathrm{Fe}^{10+}$, respectively, to establish time-averaged mean ion currents of $\approx 25 \mu \mathrm{A}$ in the storage ring during the active data collection phase. After the injection phase, continuous electron cooling was applied for $4.5 \mathrm{~s}$ to bring the average laboratory ion velocity $\left\langle v_{\text {ion }}\right\rangle$ to the average laboratory electron velocity $\left\langle v_{e}\right\rangle$, thereby decreasing the ion beam momentum spread to equilibrium at $\Delta p /\langle p\rangle \approx 10^{-4}$.

The stripping stages of an accelerator, using beam foil stripping targets, are known to produce ions in metastable states (Martinson \& Gaupp 1974). For Fe X, the only metastable level is the $3 s^{2} 3 p^{5}{ }^{2} P_{1 / 2}$, which decays to the ${ }^{2} P_{3 / 2}$ ground level with a lifetime of $\approx 15 \mathrm{~ms}$ (Ralchenko et al. 2006). Within the initial $4.5 \mathrm{~s}$ cooling period after injection and before the energy scans were started, the population of the Fe $\mathrm{x}$ metastable level 
decayed to an insignificant fraction. Fe XI in its $3 s^{2} 3 p^{4}$ ground configuration has ${ }^{3} P_{1},{ }^{3} P_{0},{ }^{1} D_{2}$, and ${ }^{1} S_{0}$ metastable levels lying above the ${ }^{3} P_{2}$ ground level. The ${ }^{3} P_{0}$ level has a lifetime of about $4 \mathrm{~s}$; the lifetimes of the other metastable levels are at least an order of magnitude shorter (Ralchenko et al. 2006). Assuming that the ion beam is purely in the $3 s^{2} 3 p^{4}$ configuration and each level is populated with a Boltzmann distribution, we have modeled the decay chain network to derive the evolution of the relative populations during the course of the measurement. Using this model and taking into account the ecool-stacking injection pattern, the calculated metastable fraction is below $2 \%$ at the beginning of the measurement energy scan. Scaling the MCBP calculations for the ${ }^{3} P_{0}$ metastable level down by the population ratio indicates that there are no significant resonances expected in our measured MBRRC spectrum. The MCBP PRRC calculations for the metastable level are $\approx 15 \%$ smaller than the PRRC ground state results in the photoionized regime and $\approx 35 \%$ smaller in the collisionally ionized regime. Scaling these down by the population ratio gives a smaller than $1 \%$ error in our experimentally derived PRRC for the ground state. Thus, the largest uncertainty from the metastable fraction is the uncertainty in the ground state population of the ion beam due to the possible $2 \%$ contamination of beam. We treat this as a systematic uncertainty which we add in quadrature to the total experimental error.

The total energy range studied was subdivided into multiple energy intervals and measured in separate runs. The energy range of each interval was selected to overlap in the laboratory frame by $50 \%$ with adjacent energy scans. In total, the electron lab energy was varied for Fe $\mathrm{x}$ from 857.7 to $1584.1 \mathrm{eV}$ and for Fe XI from 1098.9 to $1823.3 \mathrm{eV}$, with the electrons matching the velocity of the ions at an energy $E\left(v_{\text {rel }}=0\right) \approx 915 \mathrm{eV}$ for Fe $\mathrm{X}$ and $\approx 1132 \mathrm{eV}$ for Fe $\mathrm{XI}$. The lab energy intervals were subdivided into 800 steps with a stepsize of $0.122 \mathrm{eV}$. These were scanned in a wobble mode, where each measurement step of the scanning electron beam energy was followed by a reference step at a fixed energy. Slew rates in associated power supplies were taken care of by an additional $5 \mathrm{~ms}$ waiting time before beginning the data collection after each energy jump. Data were then collected at each step for $10 \mathrm{~ms}$. Within $24 \mathrm{~s}$ the energy scan was completed and a new cycle started over with a new injection phase. A run required $1 \mathrm{hr}$ on average to accumulate sufficient counting statistics. A total of 19 (14) individual runs for $\mathrm{Fe} \times$ ( $\mathrm{Fe} \mathrm{XI}$ ) were collected, covering the relative collision energy range from 0 to about $92(83) \mathrm{eV}$.

The reference step serves as a measurement of the background rate primarily due to electron capture from the residual gas. Reference points have been chosen in flat, unstructured regions of the cross section near the energy scan interval, to suppress artifacts introduced by large energy jumps. Despite these precautions, the background level still varied between overlapping runs. We derived and subtracted these offsets by successively comparing the integrated MBRRC signal in the overlapping regions of neighboring runs. The associated statistical uncertainties in the integrated signal contribute to the error budget. We began with the runs spanning across the highest observed DR series limits, using center-of-mass reference energies at $E_{\text {ref }}=73.19 \mathrm{eV}$ for Fe $\mathrm{X}$ and $70.64 \mathrm{eV}$ for Fe XI, as no DR signal was seen or is expected at these energies. The background above the series limits for these runs was shifted by adding the appropriate amount to match the small RR signal, as given by the calculations of Badnell (2006b) and modified to take the experimental field ionization into account.
The relative collision energy is established by a transformation to the center-of-mass frame described by Kilgus et al. (1992). Precise knowledge of the laboratory electron energy $E_{e}$ and ion energy $E_{i}$ is necessary. The electron energy is measured with a highly sensitive HV probe and corrected for space charge effects in the electron beam, analogous to Kilgus et al. (1992). The ion energy is defined by the electron cooling condition $\left\langle v_{i}\right\rangle=\left\langle v_{e}^{\text {cool }}\right\rangle$. The electron energy at cooling, $E_{e}^{\text {cool }}$ is inferred from the constraint that the center-of-mass MBRRC spectrum scanned for $v_{e}<v_{i}$ and $v_{e}>v_{i}$ must be symmetric around $v_{e}=v_{i}$. From such measurements, we determined $E_{e}^{\text {cool }}=$ 914.70 (3) eV for Fe x and 1131.57 (3) eV for Fe XI. From here on and throughout this paper, a quantity in parentheses specifies the uncertainty on the last significant digit(s). All errors are given at a confidence interval of $90 \%$, unless otherwise noted.

The resulting center-of-mass energy scale was linearly adjusted by a small correction factor to match the theoretical calculations. To derive the calibration factors, we selected distinct high- $n$ Rydberg resonances with narrow structure and sufficiently clean surroundings and scaled the experimental energy axis to match the theoretical MBRRC energy structure. For Fe X, we used the peak at $57.4 \mathrm{eV}$, attributed to the $3 s^{2} 3 p^{4}\left({ }^{3} P\right) 3 d 10 l$ resonance in the Fe IX product spectrum. For Fe XI, we used the peak at $49.05 \mathrm{eV}$, attributed to the $3 s^{2} 3 p^{3}\left({ }^{2} D^{\circ}\right) 3 d 9 l$ resonance in the $\mathrm{Fe} x$ product spectrum. Based on these, we calibrated the energy axis for Fe $\mathrm{x}$ by scaling it up with a factor of 1.0006 (6) and for Fe XI by 1.0065 (7).

The experimental energy distribution is best described as a flattened Maxwellian distribution (Kilgus et al. 1992) which is characterized by the longitudinal and transverse temperatures $k_{B} T_{\|}$and $k_{B} T_{\perp}$. The experimental resolution $\Delta \hat{E}$ at collision energy $\hat{E}$ is approximately given by $\Delta \hat{E}=\left[\left(\ln (2) k_{B} T_{\perp}\right)^{2}+\right.$ $\left.16 \ln (2) \hat{E} k_{B} T_{\|}\right]^{1 / 2}$ (Müller 1999). To reduce $k_{B} T_{\perp}$, the target electron beam was adiabatically expanded (Pastuszka et al. 1996) by a factor of 30 . The transverse temperature derived from fitting the measured DR spectrum was $k_{B} T_{\perp}^{\text {fit }}=3.6$ (3) $\mathrm{meV}$ for both ion species (see Section 5.1 for details on the fitting). This is consistent with expectations of $k_{B} T_{\perp}=3.6(1) \mathrm{meV}$ for thermal emission from a cathode with temperature $k_{B} T_{\text {cath }} \approx$ $110(5) \mathrm{meV}$ and an adiabatic transverse expansion factor $\xi_{e}=30$. The longitudinal temperature was derived from the width of distinct high- $n$ resonance features in the Rydberg series which yields $k_{B} T_{\|}=38(1) \mu \mathrm{eV}$. With these temperatures, the experimental energy spread for both ions amounts to $\Delta \hat{E} \approx$ $0.003 \mathrm{eV}$ at a collision energy of $\hat{E}=0.001 \mathrm{eV}, 0.005 \mathrm{eV}$ at $0.01 \mathrm{eV}, 0.009 \mathrm{eV}$ at $0.1 \mathrm{eV}, 0.023 \mathrm{eV}$ at $1 \mathrm{eV}, 0.067 \mathrm{eV}$ at $10 \mathrm{eV}$, and $0.21 \mathrm{eV}$ at $100 \mathrm{eV}$.

The dominant systematic uncertainties in the experimental results arise from the ion current measurement and the length of the interaction region and are estimated to be $25 \%$ (Lampert et al. 1996). The alignment of the electron beam angle and perpendicular position with regard to the ion beam axis has an uncertainty of below $1 \mathrm{~mm}$ throughout the $\approx 1.5 \mathrm{~m}$ long interaction zone. With an expansion factor of $\xi_{e}=30$, the electron beam has a diameter of $9 \mathrm{~mm}$. Thus, the $\approx 1 \mathrm{~mm}$ wide ion beam is fully embedded within the electron beam in this region. An uncertainty of $3 \%$ arises from variations in the transverse density profile of the probe electron beam. For Fe XI, we add $2 \%$ to account for the possible ${ }^{3} P_{0}$ metastable fraction. Adding all contributions in quadrature yields an estimated total uncertainty of $25 \%$ for both ions for the MBRRC. Another contribution to the error budget results from corrections to the 


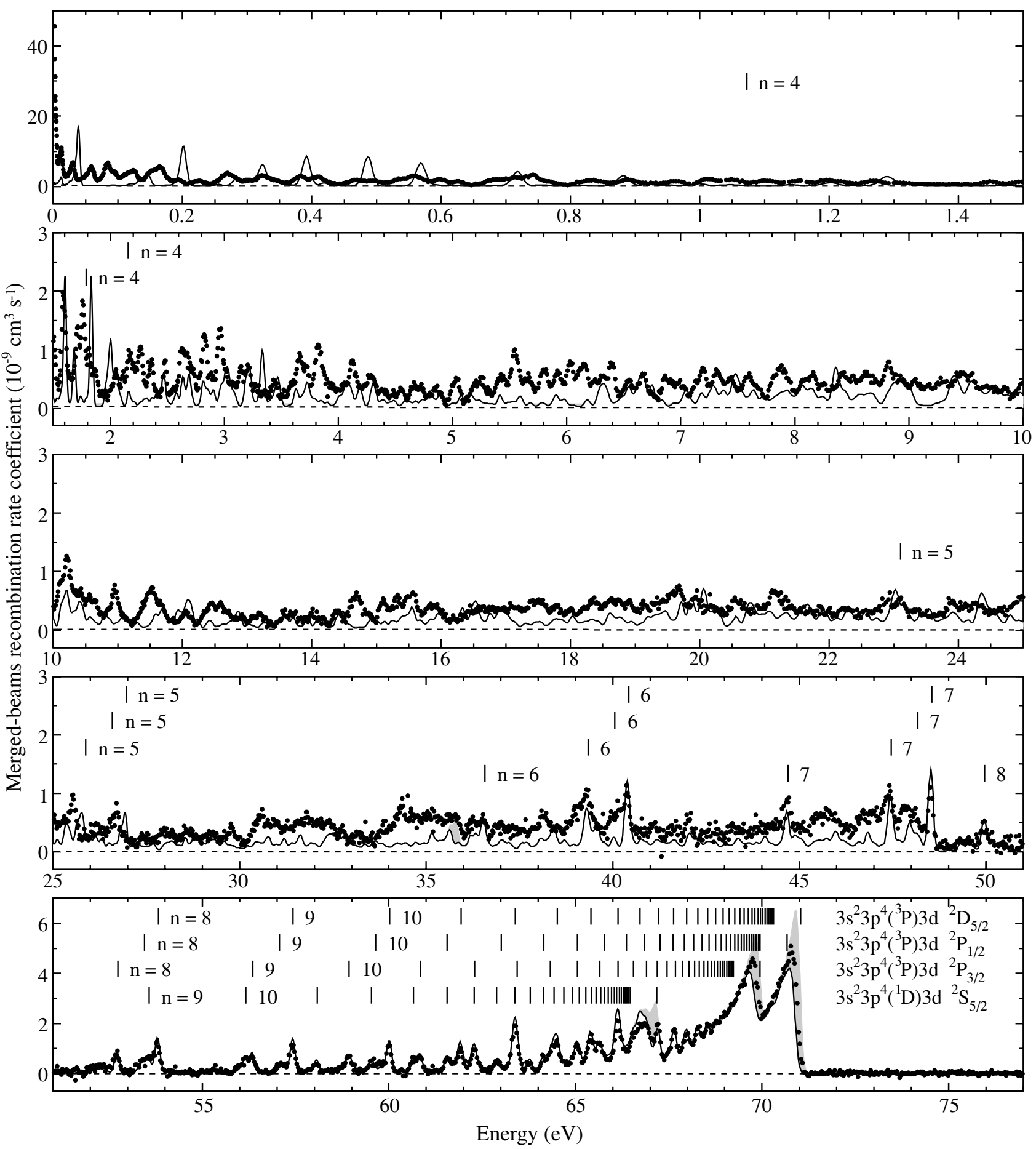

Figure 1. Fe $x$ MBRRC as a function of relative energy. The experimental MBRRC data are shown with filled circles. Our AUTOSTRUCTURE calculations including field ionization are shown by the solid line. The additional theoretical high- $n$ contributions missing from the experimental data due to field ionization are shown by the gray shaded areas. The theoretical rate coefficient has been scaled down by a uniform factor of 1.46 (see Section 4.1). The vertical lines indicate the most clearly visible Rydberg series and are labeled by the corresponding core excitation. See the text for details.

nonresonant background due to the shifting of the background level between scans. This uncertainty in the shift amounts to less than $2.7 \times 10^{-11} \mathrm{~cm}^{3} \mathrm{~s}^{-1}$ for Fe $\mathrm{x}$ (corresponding to an average of $8 \%$ of the integrated MBRRC in the energy range from 2 to $60 \mathrm{eV}$ ). For Fe XI, it is below $1.2 \times 10^{-11} \mathrm{~cm}^{3} \mathrm{~s}^{-1}$ or $3 \%$ in this range. The contributions are insignificant outside this interval. For the derived Maxwellian PRRC, this background correction when integrated with a Maxwellian distribution is insignificant at low temperatures and less than $8 \%(3 \%)$ for $\mathrm{Fe} \mathrm{X}(\mathrm{Fe} \mathrm{XI})$ at temperatures above $10^{5} \mathrm{~K}$.

\section{EXPERIMENTAL AND THEORETICAL RESULTS}

Figures 1 and 2 show the experimentally obtained MBRRC for Fe $\mathrm{X}$ and $\mathrm{Fe} \mathrm{XI}$ as a function of collision energy (circles). Also shown are the results of our DR+RR MCBP calculations using AUTOSTRUCTURE. These cross section calculations have been transformed into an MBRRC by convolving them with the relative velocity and the flattened, double-Maxwellian electron velocity distribution of the experiment using the thermal spreads derived from the measured data. The solid line shows the 

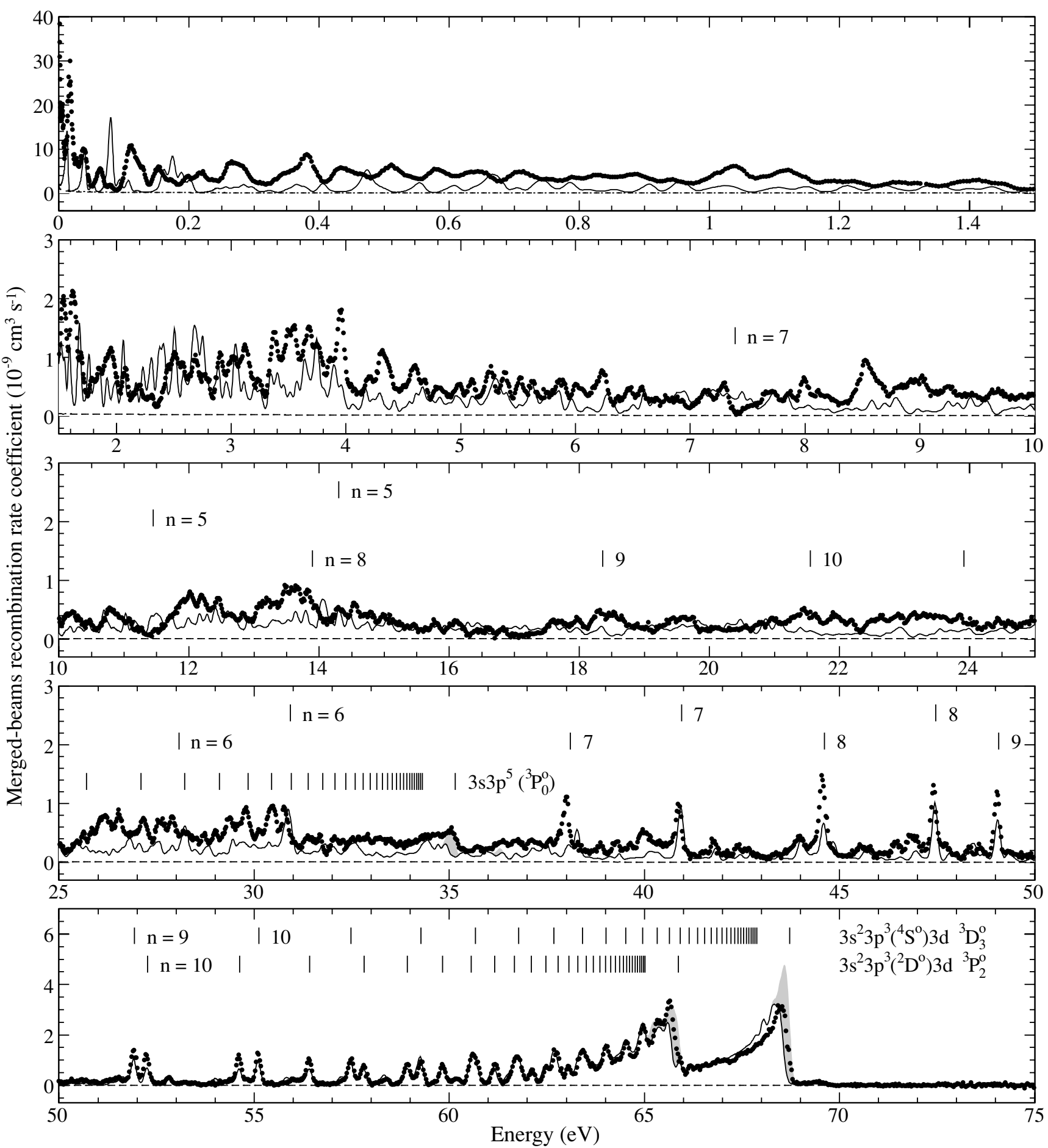

Figure 2. Same as Figure 1 but for Fe XI. Here, the theoretical rate coefficient has been scaled down by a uniform factor of 2.00 (see Section 4.2).

calculated MBRRC results taking into account the $(n, l)$-specific modifications due to field ionization, as discussed in more detail in Section 5.1. The gray areas indicate the contributions for capture into Rydberg states up to $n=1000$ which are missing from storage-ring results due to field ionization. The dashed lines show the RR only contributions (Badnell 2006b), convolved with the experimental resolution and modified by the field ionization model.

Vertical lines are used in Figures 1 and 2 to indicate the energy position of resonances from the dominant Rydberg series. These resonance energy sequences have been calculated up to $n_{\text {cut }}$ using Equation (1) and a hydrogenic approximation for the binding energy $E_{b}$. A final line is drawn at the energy position of the $n=\infty$ limit. The series limits $\Delta E$ for each of the respective series for $\mathrm{Fe} \mathrm{x}$ and $\mathrm{Fe} \mathrm{XI}$ were taken from Tables 1 and 2, respectively, and are labeled by the corresponding core excitation level.

\subsection{Fe x Merged Beams Recombination Rate Coefficients}

The Fe $\mathrm{x}$ spectrum is rich in DR features. At energies from 0 to $49.7 \mathrm{eV}$, we observe broad, unresolved structure, which can 
Table 3

Integrated DR Rate Coefficients for Fe x. The Given Errors are Purely Statistical

\begin{tabular}{lcll}
\hline $\begin{array}{l}\text { Energy Range } \\
(\mathrm{eV})\end{array}$ & $\begin{array}{c}\int \alpha_{\text {theo }} d E \\
\left(\mathrm{~cm}^{3} \mathrm{~s}^{-1} \mathrm{eV}\right)\end{array}$ & \multicolumn{1}{c}{$\begin{array}{c}\int \alpha_{\exp } d E \\
\left(\mathrm{~cm}^{3} \mathrm{~s}^{-1} \mathrm{eV}\right)\end{array}$} & $\kappa=\frac{\int \alpha_{\text {theo }} d E}{\int \alpha_{\text {exp }} d E}$ \\
\hline $0.003-0.5$ & $1.2087 \times 10^{-9}$ & $1.25(15) \times 10^{-9}$ & $0.97(12)$ \\
$0.5-2$ & $1.1205 \times 10^{-9}$ & $1.67(5) \times 10^{-9}$ & $0.67(2)$ \\
$2-10$ & $2.4802 \times 10^{-9}$ & $3.59(2) \times 10^{-9}$ & $0.691(3)$ \\
$10-36$ & $7.4894 \times 10^{-9}$ & $9.83(1) \times 10^{-9}$ & $0.7619(8)$ \\
$36-48.7$ & $4.3439 \times 10^{-9}$ & $6.192(3) \times 10^{-9}$ & $0.7015(3)$ \\
$48.7-66$ & $8.6780 \times 10^{-9}$ & $5.962(1) \times 10^{-9}$ & $1.4556(3)$ \\
$66-72$ & $1.5871 \times 10^{-8}$ & $1.1462(3) \times 10^{-8}$ & $1.3847(3)$ \\
\hline
\end{tabular}

be ascribed to the superposition of many DR resonances, but in this energy region it is impossible to reliably attribute the observed features to specific resonances. The agreement of our MCBP calculations with our measured DR structure is marginal. The calculated resonances barely coincide with the measured features. Still, suggestions of Rydberg series limits can be seen in the experimental data at some of the expected energies for such features, noticeably 35.9 and $48.7 \mathrm{eV}$, representing the $3 s 3 p^{6}{ }^{2} S_{1 / 2}$ and $3 s^{2} 3 p^{4}\left({ }^{3} P\right) 3 d^{4} D_{\{7 / 2, \ldots 1 / 2\}}$ core excitations, respectively (see Table 1 ).

Above $48.7 \mathrm{eV}$, the measured spectrum becomes less crowded and DR resonances can be more reliably assigned to observed peaks. Resonances due to higher excitations are particularly clear for electron capture into levels starting at $n=6$ and can be followed up to approximately $n=13$ before they blend into the characteristic cusp approaching the series limit. These resonances are associated with the $3 s^{2} 3 p^{4}\left({ }^{1} D\right) 3 d^{2} S_{5 / 2}$, $3 s^{2} 3 p^{4}\left({ }^{3} P\right) 3 d^{2} P_{\{3 / 2,1 / 2\}}$, and $3 s^{2} 3 p^{4}\left({ }^{3} P\right) 3 d^{2} D_{5 / 2}$ core excitations. The limits arising from these series are found in the experimental data at approximately $67 \mathrm{eV}, 70 \mathrm{eV}$, and $71 \mathrm{eV}$, respectively.

It is useful to compare in detail the integrated total DR resonance strengths over various energy intervals. The measured data at very low collision energies below $E \lesssim k_{B} T_{\perp}$ (here $\approx 3 \mathrm{meV}$ ) contain uncertain contributions due to $\mathrm{RR}$ rate enhancement further discussed in Section 5.1. In the energy interval from 0.003 to $0.5 \mathrm{eV}$, we find a ratio of the theoretical to the experimental strengths of $\kappa=\int \alpha_{\text {theo }} d E / \int \alpha_{\exp } d E=$ $0.97(12)$. At energies from 0.5 to 2,2 to 10,10 to 36 , and 36 to $48.7 \mathrm{eV}$, we observe a consistently stronger experimental MBRRC than predicted by theory, with similar ratios of $\kappa=$ $0.67(2), 0.69,0.76$, and 0.70 , respectively. These ratios, similar among each other for these energy ranges, carry errors significantly below the least significant digit given, unless explicitly stated. From $48.7 \mathrm{eV}$ and beyond, where the resonance structure of the calculations agrees well with the experiment, we find that the measured MBRRC is nevertheless considerably weaker than predicted by MCBP theory. The theory-to-experiment ratio amounts to $\kappa=1.46$ in the range of $48.7-66 \mathrm{eV}$ and to $\kappa=1.38$ at $66-72 \mathrm{eV}$; as the latter range spans across three pronounced series limits, the theoretical data there are also sensitive to the uncertainties contained in the modeling of the field ionization effect. Integrated resonance strengths for the various energy ranges are given in Table 3. In the comparison of Figure 1 and for estimating the high-Rydberg contributions unobserved because of field ionization, we use theory scaled down by $\kappa$ from the range of 48.7-66 eV, yielding good match for the lower Rydberg resonances.
Table 4

Same as Table 3 but for Fe XI

\begin{tabular}{lcll}
\hline \hline $\begin{array}{l}\text { Energy Range } \\
(\mathrm{eV})\end{array}$ & $\begin{array}{c}\int \alpha_{\text {theo }} d E \\
\left(\mathrm{~cm}^{3} \mathrm{~s}^{-1} \mathrm{eV}\right)\end{array}$ & \multicolumn{1}{c}{$\begin{array}{c}\int \alpha_{\text {exp }} d E \\
\left(\mathrm{~cm}^{3} \mathrm{~s}^{-1} \mathrm{eV}\right)\end{array}$} & $\kappa=\frac{\int \alpha_{\text {theo }} d E}{\int \alpha_{\text {exp }} d E}$ \\
\hline $0.003-0.5$ & $1.7762 \times 10^{-9}$ & $2.39(13) \times 10^{-9}$ & $0.74(4)$ \\
$0.5-2$ & $2.7179 \times 10^{-9}$ & $3.83(8) \times 10^{-9}$ & $0.71(2)$ \\
$2-5$ & $2.7658 \times 10^{-9}$ & $2.18(3) \times 10^{-9}$ & $1.27(2)$ \\
$5-10.2$ & $2.0254 \times 10^{-9}$ & $2.04(1) \times 10^{-9}$ & $0.993(5)$ \\
$10.2-37.2$ & $1.0569 \times 10^{-8}$ & $1.0090(2) \times 10^{-8}$ & $1.0474(2)$ \\
$37.2-55.5$ & $5.5325 \times 10^{-9}$ & $5.158(2) \times 10^{-9}$ & $1.0726(3)$ \\
$55.5-60.2$ & $1.0341 \times 10^{-8}$ & $5.1761(3) \times 10^{-9}$ & $1.9979(2)$ \\
$60.2-75$ & $1.0633 \times 10^{-8}$ & $5.936(3) \times 10^{-9}$ & $1.791(1)$ \\
\hline
\end{tabular}

\subsection{Fe XI Merged Beams Recombination Rate Coefficients}

The Fe XI DR spectrum is as rich as that of Fe X. Below about $35 \mathrm{eV}$, no interval can be found where the experimental spectrum is free of DR contributions and broad, unresolved structure dominates the spectrum. Again, we find in this range poor agreement between our measurement and our theoretical calculation of the resonance structure. Near $35.1 \mathrm{eV}$ a weak series limit can be identified, showing a broad, unstructured cusp in the DR signal. This is attributed to DR via $3 s 3 p^{4}\left({ }^{3} P_{0}^{o}\right)$ core excitation (see Table 2). Yet, we are unable to unambiguously identify any individual resonance features due to this series.

Above $35 \mathrm{eV}$ the measured spectrum becomes less crowded and DR resonances can be assigned to observed peaks with more confidence. DR resonances via $3 s^{2} 3 p^{3}\left({ }^{2} D^{o}\right) 3 d^{3} P_{2}^{o}$ and $3 s^{2} 3 p^{3}\left({ }^{4} S^{o}\right) 3 d^{3} D_{3}^{o}$ core excitations can be clearly identified, with series limits at $65.9 \mathrm{eV}$ and $68.7 \mathrm{eV}$, respectively. Distinct resonance features due to these two series can be traced for capture into Rydberg levels from $n=7$ to $n \approx 17$ before the resolving power of the experiment blends them into one another. For the $n=5$ and 6 levels of these series, unambiguous identification of the corresponding peaks in the measured spectrum is not possible due to a combination of fine structure splitting and resonances from other series. At $69.7 \mathrm{eV}$ another small cusp in the data can be found which can be attributed to $3 s^{2} 3 p^{3}\left({ }^{4} S^{o}\right) 3 d^{3} D_{2}^{o}$ core excitation.

Comparing in detail the integrated theoretical and experimental resonance strengths for DR over various energy intervals, we find a behavior different from what was found for Fe $\mathrm{X}$, with the integrated resonance strengths and the theory-to-experiment ratios $\kappa$ listed in Table 4 . Also here, at lower energies theory generally underestimates the experimental MBRRC by up to about $30 \%$, while it overestimates it by up to $100 \%$ along the upper part of the dominant Rydberg series. In the comparison of Figure 2 and for estimating the high Rydberg contributions unobserved because of field ionization, we use theory scaled down by $\kappa=2$ from the range of $55.5-65 \mathrm{eV}$.

\section{PLASMA DR RATE COEFFICIENTS}

\subsection{Derivation}

The derivation of a plasma rate coefficient from the experimental MBRRC data requires accounting for three issues relating to the measurement technique. These are broadening of resonances due the experimental resolution (Schippers et al. 2004), low-energy enhancement of the RR signal (Gwinner et al. 2000; Hörndl et al. 2006), and field ionization of high- $n$ Rydberg levels. In order to address these issues, we split the measured MBRRC spectrum into two parts, as has been described more 

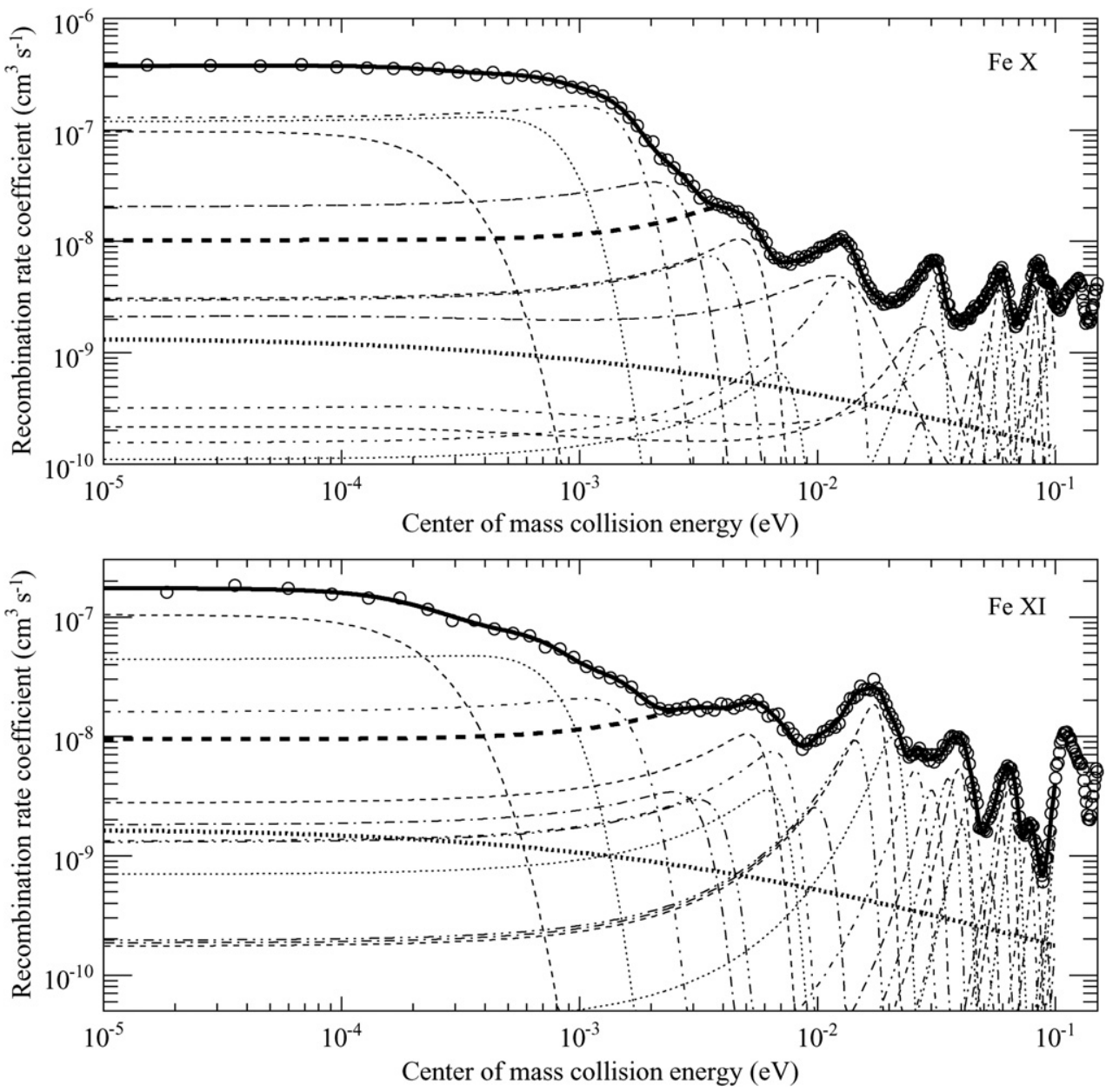

Figure 3. Fitted MBRRC model spectrum in the energy range from $10^{-5}$ to $0.1 \mathrm{eV}$ for both Fe $\mathrm{x}$ (upper panel) and Fe XI (lower panel). The open circles show the measured data. The thick solid line shows the convolved fitted model spectrum $\left\langle v \sigma_{\mathrm{lo}}^{\mathrm{DR}}(E)\right\rangle+\left\langle v \sigma^{\mathrm{RR}}(E)\right\rangle$. The contributions from the individual resonances in the total model spectrum are indicated using thin lines and varying line style. The thick dashed line gives the modified fit, where resonances below 3 meV have been excluded. $\mathrm{RR}$ is shown with a thick dotted line.

comprehensively in the work of Schmidt et al. (2008): one below $0.1 \mathrm{eV}$ and one above.

For the measured data in the interval below $0.1 \mathrm{eV}$, the DR PRRC is derived from an empirical model cross section spectrum $\sigma_{\mathrm{lo}}^{\mathrm{DR}}(E)$ fitted to the measured MBRRC. This model spectrum is convolved with the relative velocity and a flattened, double-Maxwellian electron velocity distribution (Kilgus et al. 1992). The transverse and longitudinal temperatures, as well as the resonance energies and strengths are free parameters in this fit. The measured spectrum is a blend of an unknown number of resonances which we model using a mixture of $\delta$-functions and Lorentzian line shapes. For Fe X, our model includes 21 resonances between 0 and $0.1 \mathrm{eV}$. For Fe XI, the model uses 24 resonances in this energy range. A few additional resonances just above the high end of the fitting interval are included to ensure the continuity of the fit with the measured data. The curves for the total fit and for each resonance are shown in Figure 3. Calculation of the RR cross section $\sigma_{\mathrm{RR}}(E)$ is modeled using the hydrogenic Bethe-Salpeter description (Hoffknecht et al. 2001). We scale the convolved RR rate coefficient $\alpha_{\mathrm{RR}}(E)$ to the results of the more sophisticated AUTOSTRUCTURE calculations by Badnell (2006b). The deviation of the scaled Bethe-Salpeter curve from the AUTOSTRUCTURE results is less than 1.5\% in the fitted energy interval. Since these nonenhanced RR contributions (see below) account for only about $1 \%$ of the total MBRRC signal at $0 \mathrm{eV}$ relative energy, this deviation can be neglected in the error budget.

In merged electron-ion beam experiments using magnetically guided electron beams, one typically finds an enhanced MBRRC at relative energy of the beams in a range starting below the transverse electron energy $k_{B} T_{\perp}$, with $k_{B} T_{\perp} \approx 3 \mathrm{meV}$ in the present experiment. This is caused by the motionally induced electric fields generated by the toroidal magnetic fields in the merging and demerging regions of the electron coolers. Gwinner et al. (2000) and Hörndl et al. (2006) have shown dependences on the electron beam temperature, ion species, and the specific geometry of the electron beam setup. Hörndl et al. (2006) showed that the enhancement in general becomes visible at energies $E \lesssim k_{B} T_{\perp}$. For measurements using the TSR electron cooler, typical enhancement ratios in the range of $\left[\Delta \alpha(E) / \alpha_{0}(E)\right]+1=1.5-3$ (Wolf \& Gwinner 2003) were observed, where $\alpha_{0}(E)$ is the theoretically expected value and $\Delta \alpha(E)$ is the contribution due to enhancement (for details see Gwinner et al. 2000). This uncertainty in the low-energy recombination signal at interaction energies in the range below $k_{B} T_{\perp}$ is inherent to our method, however, it does not contribute to the recombination signal in "natural" astrophysical plasmas and hence needs to be removed before deriving a true PRRC.

Our modeling does not account for the RR enhancement and is thus likely to overestimate the strengths of the DR 


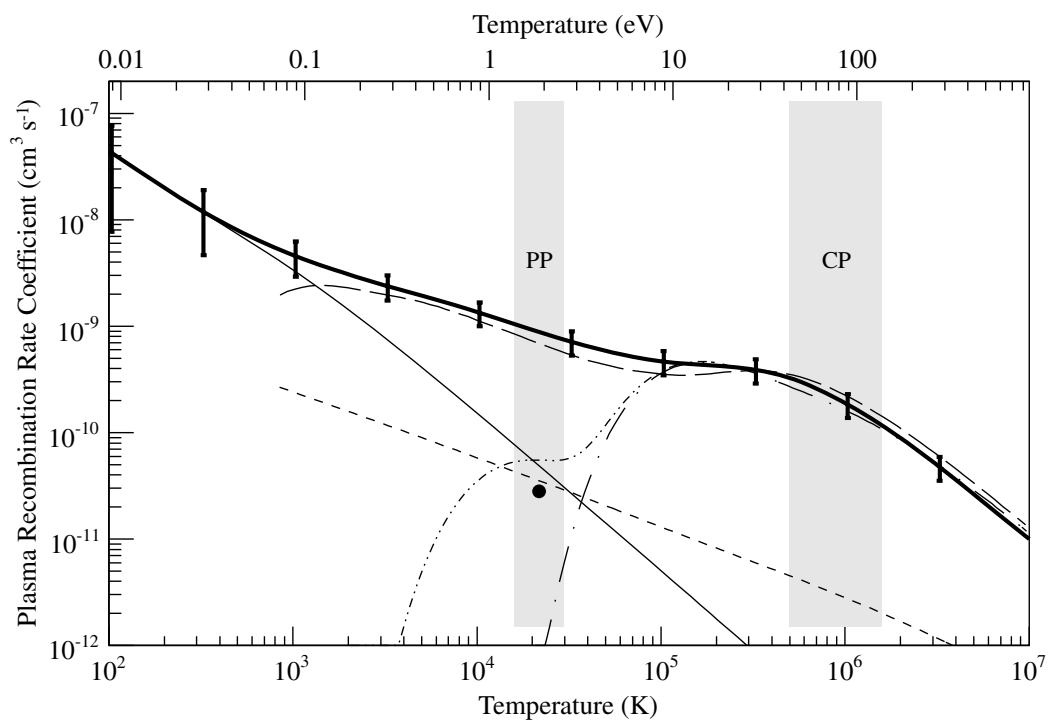

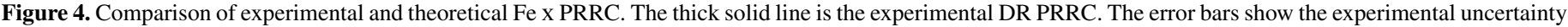

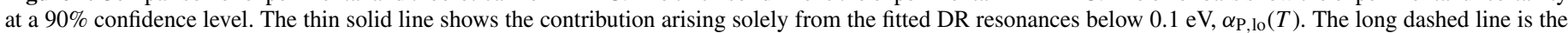

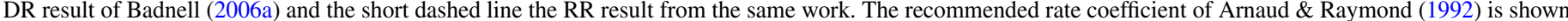

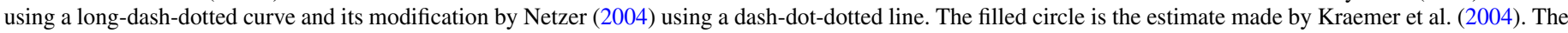

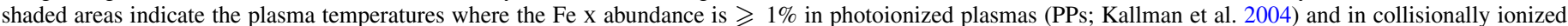
plasmas (CPs; Bryans et al. 2006).

resonances at $E \lesssim k_{B} T_{\perp}$, corresponding to a range below $3 \mathrm{meV}$ in the present experiment. For assessing the possible systematic errors due to this uncertainty at the lowest merged beam collision energies, we consider our results for two cases to derive an estimated upper limit and a hard lower limit for the DR contributions to $\alpha_{\text {exp }}(E)$ : in the first case, we keep all 21 (24) fitted resonances for $\mathrm{Fe} \mathrm{x}(\mathrm{Fe} \mathrm{XI})$, in the second case we remove the 4 (3) resonances below $3 \mathrm{meV}$ for $\mathrm{Fe} \mathrm{x}(\mathrm{Fe} \mathrm{XI})$ from the model cross section spectrum. Comparing the convolved model for the second case (thick dashed line in Figure 3) to the measured data, at $0.01 \mathrm{meV}$ we find ratios of $\alpha_{\text {exp }}(E) / \alpha_{\text {fit }}^{\text {wo }}(E) \approx 36.6$ for Fe $\mathrm{x}$ and $\approx 18.3$ for Fe XI as upper limits for the rate enhancement. These enhancements are more than an order of magnitude larger than those determined for the cooler. For the electron target, a preliminary study of the rate enhancement found an enhancement ratio of $\approx 4.6$ for Li-like F VII (Lestinsky 2007). Schmidt et al. (2008) also carried out measurements using the electron target. They reported enhancement factors of 7.5 and 3.7 for Fe VIII and Fe IX, respectively. Thus, the amount of observed enhancement here strongly suggests the presence of unresolved DR resonances below $3 \mathrm{meV}$.

We derive DR plasma rate coefficients $\alpha_{\mathrm{P}, \mathrm{lo}_{\mathrm{o}}}^{\mathrm{wi}}(T)$ and $\alpha_{\mathrm{P}, \mathrm{lo}_{\mathrm{o}}}^{\mathrm{wo}}(T)$ by convolving the respective model DR cross sections (with and without those resonances below $3 \mathrm{meV}$, respectively) with the relative velocity and an isotropic Maxwellian electron energy distribution (Schippers et al. 2001). The average of $\alpha_{\mathrm{P}, 1 \mathrm{o}}^{\mathrm{wi}}(T)$ and $\alpha_{\mathrm{P}, 1 \mathrm{o}}^{\mathrm{wo}}(T)$ is taken as the experimentally derived plasma DR rate coefficients $\alpha_{\mathrm{P}, \mathrm{lo}}(T)$. The half-difference between both is propagated to the error budget as an experimental uncertainty associated with the rate enhancement. This error is large only at very low plasma temperatures and is unimportant for temperatures above $10^{3} \mathrm{~K}$.

Above $0.1 \mathrm{eV}$ the measured data are treated by subtracting the theoretical (small) RR from the experimental MBRRC spectrum to obtain a pure DR spectrum $\alpha_{\mathrm{DR}}(E)$. The missing high- $n$ Rydberg contributions are accounted for by replacing the experimental data $\alpha_{\mathrm{DR}}(E)$ in intervals sensitive to field ionization with the scaled theoretical MBRRC for $n \leqslant 1000$,

$$
\alpha_{\mathrm{DR}}^{\prime}(E)= \begin{cases}\alpha_{\mathrm{DR}}(E) & : 0.1 \mathrm{eV} \leqslant E \leqslant E_{\mathrm{cut}}, \\ \kappa^{-1} \alpha_{\mathrm{DR}}^{\mathrm{MCBP}}(E, n \leqslant 1000) & : E>E_{\mathrm{cut}} .\end{cases}
$$

We use $E_{\text {cut }}=64 \mathrm{eV}$ for Fe $\mathrm{x}$ and $64.2 \mathrm{eV}$ for Fe XI. The scaling factors used in this small correction are chosen as $\kappa=1.46$ and 2.00 for Fe $\mathrm{x}$ and Fe XI, respectively, as discussed in Sections 4.1 and 4.2 above. The uncertainties are below $1 \%$ and can thus be safely ignored in the error budget. From this modified MBRRC we derive a PRRC $\alpha_{\mathrm{P}, \mathrm{hi}}(T)$ by convolving the MBRRC with an isotropic Maxwellian electron energy distribution (Schippers et al. 2001). The final total DR PRRC $\alpha_{\mathrm{P}}(T)$ is the sum of $\alpha_{\mathrm{P}, \mathrm{lo}}(T)$ and $\alpha_{\mathrm{P}, \mathrm{hi}}(T)$.

\subsection{PRRC Results and Comparison}

Figures 4 and 5 show the experimentally derived DR PRRC (thick solid line) in the temperature range from $10^{2}$ to $10^{7} \mathrm{~K}$ for Fe $\mathrm{x}$ and Fe XI, respectively. The contributions from DR resonances below $0.1 \mathrm{eV}, \alpha_{\mathrm{P}, \mathrm{lo}}(T)$, are shown with a thin solid line and are less than $10 \%$ of $\alpha_{\mathrm{P}}(T)$ for both ions at temperatures relevant to their formation in PPs and negligible for CPs. For $\mathrm{Fe} \mathrm{x}$, the size of the error bars is $82 \%$ at $10^{2} \mathrm{~K}, 67 \%$ at $3.3 \times 10^{2} \mathrm{~K}, 36 \%$ at $10^{3} \mathrm{~K}, 27 \%$ at $3.3 \times 10^{3} \mathrm{~K}$, and $25 \%$ from $10^{4} \mathrm{~K}$ and up. For Fe XI, the uncertainty is $47 \%$ at $10^{2} \mathrm{~K}$, $29 \%$ at $3.3 \times 10^{2} \mathrm{~K}$, and $25 \%$ from $10^{3} \mathrm{~K}$ on. The temperature ranges where the fractional abundance of each ion is $\geqslant 1 \%$ of the total Fe abundance in PPs and in CPs are indicated as gray shaded areas (Kallman et al. 2004; Bryans et al. 2006).

Also shown in Figures 4 and 5 are the recommended DR rate coefficients of Arnaud \& Raymond (1992) plotted using a long dash-dotted line. They severely underestimate the DR PRRC of both ions for plasma temperatures below $10^{5} \mathrm{~K}$, which are of particular importance for PPs. Similar behavior was observed for other M-shell iron ions (Schmidt et al. 2006, 2008; Lukić et al. 2007). As discussed in Section 2, this discrepancy arises mostly from the fact that the underlying theoretical calculations were carried out for high-temperature plasmas. Netzer (2004) 


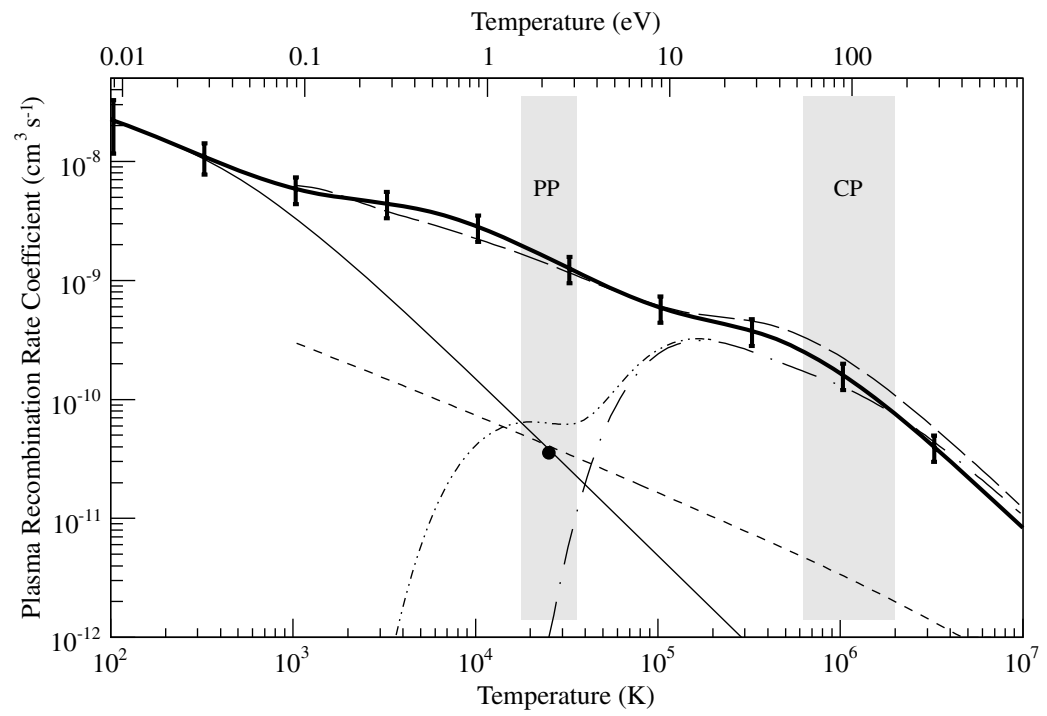

Figure 5. Same as Figure 4 but for Fe XI.

attempted to modify the results of Arnaud \& Raymond (1992) in an ad hoc manner, but as is clearly seen in Figures 4 and 5 (thin dash-double-dotted line) his guess was still too low. Kraemer et al. (2004) presented a guess for the plasma rate coefficients for Fe M-shell ions based on results from L-shell C, N, O, and $\mathrm{Fe}$ ions. Their results (solid circle) are also too low.

The most recent theoretical DR rate coefficients by Badnell (2006b) are shown in Figures 4 and 5 by the long dashed line. As discussed in Section 2, the calculations carried out in the frame of the present work result in PRRC insignificantly different from those of Badnell (2006b). The RR rate coefficients from the same work are drawn with a short dashed line. These results were specified only for the temperature range of $\left(10^{1}-10^{7}\right) \times z^{2} \mathrm{~K}$, where $z$ is the parent ion charge state. These recent calculations are in reasonable agreement with our experimentally derived PRRCs, though some discrepancies with the experimental data can still be observed. For Fe X, theory differs from experiment by factors of $0.80-0.76$ for PP and by $1.08-1.22$ for CP. Here and below in each pair of numbers quoted, the first corresponds to the lower temperature limit and the second to the higher temperature limit of the cited formation zone. These differences are within the experimental uncertainties. For Fe XI, we find factors of $0.85-0.93$ for PPs and 1.33-1.42 for collisionally ionized plasmas. The deviation at high temperatures arises mostly from the larger theoretical resonance strengths relative to the experiment toward the series limits (see Section 4.2). For both ions, the reasonable agreement between experimental and theoretical DR plasma rate coefficients is surprising considering the poor agreement between the measured and predicted MBRRC resonance structure.

\subsection{Parameterization of Plasma Rate Coefficients}

We have parameterized our experimentally derived DR PRRCs $\alpha_{\mathrm{P}}(T)$ by fitting the function

$$
\alpha_{\mathrm{P}}^{\mathrm{fit}}(T)=T^{-3 / 2} \sum_{i} c_{i} \exp \left(-E_{i} / T\right)
$$

to $\alpha_{\mathrm{P}}(T)$. The fitting results for the free parameters $c_{i}$ and $E_{i}$ are given in Table 5 for both ions. These fits are valid for the energy range of $10^{2}-10^{7} \mathrm{~K}$, the residuals $R=\left[\alpha_{\mathrm{P}}(T)-\alpha_{\mathrm{P}}^{\mathrm{fit}}(T)\right] / \alpha_{\mathrm{P}}(T)$, are less than $\pm 3 \%$ for temperatures between $10^{2}$ and $10^{5} \mathrm{~K}$ and below $\pm 1 \%$ above.
Table 5

Fit Parameters $c_{i}\left(\mathrm{~cm}^{3} \mathrm{~s}^{-1} \mathrm{~K}^{3 / 2}\right)$ and $E_{i}(\mathrm{~K})$ for the Experimental DR PRRC for $\mathrm{Fe} \mathrm{x}$ and $\mathrm{Fe} \mathrm{XI}$ Using Equation (5)

\begin{tabular}{ccccc}
\hline \hline & \multicolumn{2}{c}{ Fe X } & \multicolumn{2}{c}{ Fe XI } \\
$i$ & $c_{i}$ & $E_{i}$ & $c_{i}$ & $E_{i}$ \\
\hline 1 & $6.485(-5)$ & $3.994(1)$ & $6.487(-5)$ & $1.101(2)$ \\
2 & $6.360(-5)$ & $5.621(2)$ & $8.793(-5)$ & $5.654(2)$ \\
3 & $3.720(-4)$ & $1.992(3)$ & $4.939(-4)$ & $1.842(3)$ \\
4 & $1.607(-3)$ & $8.325(3)$ & $3.787(-3)$ & $7.134(3)$ \\
5 & $3.516(-3)$ & $2.757(4)$ & $8.878(-3)$ & $3.085(4)$ \\
6 & $7.326(-3)$ & $7.409(4)$ & $5.325(-2)$ & $1.878(5)$ \\
7 & $2.560(-2)$ & $1.552(5)$ & $2.104(-1)$ & $6.706(5)$ \\
8 & $1.005(-1)$ & $4.388(5)$ & $\ldots$ & $\ldots$ \\
9 & $1.942(-1)$ & $7.355(5)$ & $\ldots$ & $\ldots$ \\
\hline
\end{tabular}

Note. Here, the notation $x(y)$ denotes $x \times 10^{y}$.

\section{SUMMARY}

We have measured the MBRRC for Fe $\mathrm{x}$ and $\mathrm{Fe} \mathrm{XI}$ at the TSR heavy-ion storage ring. The MCBP theory of Badnell (2006b) and our experimental DR results for $\mathrm{Fe} \mathrm{X}(\mathrm{Fe} \mathrm{XI})$ are in poor agreement with regards to the resonance structure for energies below $48(35) \mathrm{eV}$. Better agreement is found with the integrated resonance strength over this energy range. Above 48 (35) eV we find good agreement for the resonance structure but poor agreement for the integrated resonance strengths with theory being larger than experiment. Our results suggest that more specific theoretical quantities such as DR-generated spectral line excitations could be considerably uncertain. The origin of these deviations, which have already been observed in previous $\mathrm{Fe}$ M-shell studies (Schmidt et al. 2006; Lukić et al. 2007; Schmidt et al. 2008), remains unknown.

From our measured data, we extracted the DR contributions and transformed them into a DR PRRC for each ion over a temperature range of $10^{2}-10^{7} \mathrm{~K}$. This range includes the temperatures where each ion is abundant in both photoionized and CPs. As expected, the recommended DR data of Arnaud \& Raymond (1992) underestimate the actual DR PRRC by several orders of magnitude at temperatures relevant for PPs. This continues a trend from our earlier measurements for other Fe M-shell ions (Schmidt et al. 2006, 2008; Lukić et al. 2007). The ad hoc estimates by Netzer (2004) and Kraemer et al. (2004) 
turn out to be still too low. Despite the differences between experiment and state-of-the-art MCBP theory, the MCBP PRRC for each ion agrees surprisingly well with the experimentally derived value. The varying ratios of theoretical to experimental integrated resonance strengths tend to cancel each other due to the temperature averaging of the PRRC, producing reasonable agreement over a wide range of temperatures. For photoionized gas, the results for both ions agree within the uncertainty limits. For collisionally ionized gas, the theoretical PRRC results have a tendency to be somewhat larger than the experimentally derived results.

We appreciate the efficient support by the accelerator and TSR group during the beam time. M.L. thanks Elmar Träbert for the discussion of metastable populations. This work was supported by the German federal research funding agency DFG under contract no. Schi 387/5. M.L., D.V.L. and D.W.S. were supported in part by the NASA Astronomy and Physics Research and Analysis program, and the Solar and Heliospheric Physics program.

\section{APPENDIX}

\section{LIST OF ABBREVIATIONS}

$\begin{array}{ll}\text { AGN } & \text { active galactic nucleus } \\ \text { CP } & \text { collisionally ionized plasma } \\ \text { DR } & \text { dielectronic recombination } \\ \text { MCBP } & \text { multiconfiguration Breit-Pauli } \\ \text { MBRRC } & \text { merged beams recombination rate coefficient } \\ \text { PP } & \text { photoionized plasma } \\ \text { PRRC } & \text { plasma rate coefficient } \\ \text { RR } & \text { radiative recombination } \\ \text { UTA } & \text { unresolved transition array }\end{array}$

\section{REFERENCES}

Altun, Z., Yumak, A., Badnell, N. R., Loch, S. D., \& Pindzola, M. S. 2006, A\&A, 447, 1165

Altun, Z., Yumak, A., Yavuz, I., Badnell, N. R., Loch, S. D., \& Pindzola, M. S. 2007, A\&A, 474, 1051

Arnaud, M., \& Raymond, J. 1992, ApJ, 398, 394

Badnell, N. R. 1986, J. Phys. B: At. Mol. Phys., 19, 3827

Badnell, N. R. 2006a, J. Phys. B: At. Mol. Opt. Phys., 39, 4825

Badnell, N. R. 2006b, ApJ, 651, L73

Badnell, N. R., et al. 2003, A\&A, http://amdpp.phys.strath.ac.uk/tamoc/DR, 406, 1151

Baryshevsky, V., Korzhik, M., Moroz, V., Pavlenko, V., \& Fyodorov, A. 1991, Nucl. Instrum. Methods B, 58, 291

Behar, E., Rasmussen, A. P., Blustin, A. J., Sako, M., Kahn, S. M., Kaastra, J. S., Branduardi-Raymont, G., \& Steenbrugge, K. C. 2003, ApJ, 598, 232

Behar, E., Sako, M., \& Kahn, S. M. 2001, ApJ, 563, 497

Blustin, A. J., Branduardi-Raymont, G., Behar, E., Kaastra, J. S., Kahn, S. M., Page, M. J., Sako, M., \& Steenbrugge, K. C. 2002, A\&A, 392, 453

Bryans, P., Badnell, N. R., Gorczyca, T. W., Laming, J. M., Mitthumsiri, W., \& Savin, D. W. 2006, ApJS, 167, 343

Chakravorty, S., Kembhavi, A. K., Elvis, M., Ferland, G., \& Badnell, N. R. 2008, MNRAS, 384, L24

Ferland, G. J., Korista, K. T., Verner, D. A., Ferguson, J. W., Kingdon, J. B., \& Verner, E. M. 1998, PASP, 110, 761

Gallagher, T. F. 1994, Cambridge Monographs on Atomic, Molecular, and Chemical Physics Rydberg Atoms 3: (Cambridge: Cambridge Univ. Press)

Gallo, L. C., Boller, T., Brandt, W. N., Fabian, A. C., \& Vaughan, S. 2004, A\&A, 417,29

Grieser, M., Habs, D., von Hahn, R., Kleffner, C. M., Repnow, R., Stampfer, M., Jaeschke, E., \& Steck, M. 1991, in Proc. IEEE Particle Accelerator Conf., Vol. 5, ed. L. Lizana \& J. Chew (New York: IEEE), 2817
Gu, M. F. 2003, ApJ, 590, 1131

Gu, M. F. 2004, ApJS, 153, 389

Gwinner, G., et al. 2000, Phys. Rev. Lett., 84, 4822

Habs, D., et al. 1989, Nucl. Instrum. Methods B, 43, 390

Hahn, Y. 1989, J. Quant. Spectrosc. Radiat. Transfer, 41, 315

Hess, C. J., Kahn, S. M., \& Paerels, F. B. S. 1997, ApJ, 478, 94

Hoffknecht, A., Schippers, S., Müller, A., Schwalm, D., \& Wolf, A. 2001, Phys. Scr., T92, 402

Hörndl, M., Yoshida, S., Wolf, A., Gwinner, G., Seliger, M., \& Burgdörfer, J. 2006, Phys. Rev. A, 74, 052712

Jacobs, V. L., Davis, J., Kepple, P. C., \& Blaha, M. 1977, ApJ, 211, 605

Kallman, T. R., \& Bautista, M. 2001, ApJS, 133, 221

Kallman, T. R., Palmeri, P., Bautista, M. A., Mendoza, C., \& Krolik, J. H. 2004, ApJS, 155, 675

Kaspi, S., Netzer, H., Chelouche, D., George, I. M., Nandra, K., \& Turner, T. J. 2004, ApJ, 611, 68

Kaspi, S., et al. 2002, ApJ, 574, 643

Kilgus, G., Habs, D., Schwalm, D., Wolf, A., Badnell, N. R., \& Müller, A. 1992, Phys. Rev. A, 49, 5730

Kraemer, S. B., Ferland, G. J., \& Gabel, J. R. 2004, ApJ, 604, 556

Krolik, J. H., McKee, C. F., \& Tarter, C. B. 1981, ApJ, 249, 422

Krongold, Y., Nicastro, F. M., Brickhouse, N. S., Mathura, S., \& Zezas, A. 2005, ApJ, 620, 165

Lampert, A., Wolf, A., Habs, D., Kenntner, J., Kilgus, G., Schwalm, D., Pindzola, M. S., \& Badnell, N. R. 1996, Phys. Rev. A, 53, 1413

Lestinsky, M. 2007, Ph.D. thesis, Univ. Heidelberg (http://www.ub.uniheidelberg.de/archiv/7334/)

Lestinsky, M., et al. 2008, Phys. Rev. Lett., 100, 033001

Linkemann, J., et al. 1995, Nucl. Instrum. Methods B, 98, 154

Lukić, D. V., et al. 2007, ApJ, 664, 1244

Martinson, I., \& Gaupp, A. 1974, Phys. Rep., 15, 113

Matsumoto, C., Leighly, K. M., \& Marshall, H. L. 2004, ApJ, 603, 456

McKernan, B., Yaqoob, T., \& Reynolds, C. S. 2007, MNRAS, 379, 1359

Müller, A. 1999, Int. J. Mass Spectrom., 192, 9

Müller, A. 2008, Adv. At. Mol. Opt. Phys., 55, 293

Netzer, H. 2004, ApJ, 604, 551

Netzer, H., et al. 2003, ApJ, 599, 933

Pastuszka, S., et al. 1996, Nucl. Instrum. Methods A, 369, 11

Pindzola, M. S., Badnell, N. R., \& Griffin, D. C. 1992, Phys. Rev. A, 46, 5725

Poth, H. 1990, Phys. Rep., 196, 135

Pounds, K. A., Reeves, J. N., King, A. R., \& Page, K. L. 2004, MNRAS, 350, 10

Pounds, K. A., Reeves, J. N., O’Brien, P. T., Page, K. A., Turner, M. J. L., \& Nayakshin, S. 2001, ApJ, 559, 181

Ralchenko, Y., Kramida, A., \& Reader, J. 2006, Atomic Spectra Database, version 3.1, http://physics.nist.gov/PhysRefData/ASD/index.html

Reynolds, C. S., \& Fabian, A. C. 1995, MNRAS, 273, 1167

Sako, M., et al. 2001, A\&A, 365, L168

Sako, M., et al. 2003, ApJ, 596, 114

Savin, D. W., et al. 1997, ApJ, 489, L115

Savin, D. W., et al. 1999, ApJS, 123, 687

Savin, D. W., et al. 2002a, ApJS, 138, 337

Savin, D. W., et al. 2002b, ApJ, 576, 1098

Savin, D. W., et al. 2003, ApJS, 147, 421

Savin, D. W., et al. 2006, ApJ, 642, 1275

Schippers, S. 2008, J. Phys. Conf. Ser., in press (arXiv:0808.3366)

Schippers, S., Müller, A., Gwinner, G., Linkemann, J., Saghiri, A., \& Wolf, A. 2001, ApJ, 555, 1027

Schippers, S., Schnell, M., Brandau, C., Kieslich, S., Müller, A., \& Wolf, A. 2004, A\&A, 421, 1185

Schmidt, E. W., et al. 2006, ApJ, 641, L157

Schmidt, E. W., et al. 2008, A\&A, 492, 265

Schnell, M., et al. 2003, Phys. Rev. Lett., 91, 043001

Seaton, M. J., \& Storey, P. J. 1976, in Atomic Processes and Applications, ed. P. G. Burke \& B. L. Moiseiwitsch (Amsterdam: North-Holland), 133

Sprenger, F., Lestinsky, M., Orlov, D. A., Schwalm, D., \& Wolf, A. 2004, Nucl. Instrum. Methods A, 532, 298

Steck, M., et al. 1990, Nucl. Instrum. Methods A, 287, 324

Steenbrugge, K. C., Kaastra, J. S., de Vries, C. P., \& Edelson, R. 2003, A\&A, 402, 477

Steenbrugge, K. C., Kaastra, J. S., Sako, M., Branduardi-Raymont, G., Behar, E., Paerels, F. S. B., Blustin, A. J., \& Kahn, S. M. 2005, A\&A, 432, 453

Wissler, G. 2002, Ph.D. thesis, Univ. Heidelberg (http://www.ub.uniheidelberg.de/archiv/3101)

Wolf, A., \& Gwinner, G. 2003, Hyperfine Interact., 146/147, 5

Wolf, A., et al. 2006, Hyperfine Interact., 172, 111 